\title{
Choledochal cyst in situs inversus totalis: Hitherto unreported association
}

\author{
Syed Aslam Shah, Qurrat Al Ain Atif, I ftikhar Ahmed Soomro, Muhammad Saaiq \\ Department of General Surgery, Pakistan Institute of Medical Sciences (PIMS), Islamabad, Pakistan \\ Correspondence: Syed Aslam Shah, Professor and Head of Surgery. Address: House No. 352, Street 33, Sector F-11/2 \\ Islamabad, Pakistan. Email: drsaslamshah@gmail.com
}

Received: January 19, 2014

Accepted: February 19, $2014 \quad$ Online Published: February 28, 2014

DOI : $10.5430 /$ crcp.v1n1p27

URL: http://dx.doi.org/10.5430/crcp.v1n1p27

\begin{abstract}
Situs inversus totalis is a rare autosomal recessive disorder characterized by transposition of the intra-abdominal as well as the intrathoracic viscera. We present the case of a 45-year-old Pakistani lady who had choledochal cyst with cholelithiasis in tandem with situs inversus totalis. We were faced with dilemmas both diagnostic and management during the course of her care. We share our experience with emphasis on both ultrasonologist as well as surgical team members to be mindful of the possibility of an underlying choledochal cyst when they are dealing with symptomatic gall stone disease in such patients. This will ensure appropriate surgical planning and avert the avoidable dilemmas intra-operatively.
\end{abstract}

\section{Key words}

Choledochal cyst, Situs inversus totalis, Cholelithiasis

\section{I ntroduction}

Situs inversus is a congenital anomaly characterized by transposition of the abdominal viscera. When this anomalous condition is associated with dextrocardia, it is known as situs inversus totalis. The earliest documented description of situs inversus totalis with dextrocardia is found in 1643 from the anatomist surgeon Marco Aurelio ${ }^{[1-3]}$. It is a rare condition with its incidence reported variably as $1-2 / 10,000$ normal population. The mode of transmission is autosomal recessive; however the exact etiology continues to remain elusive ${ }^{[3-5]}$.

To the best of our information, choledochal cyst with cholelithiasis has not been reported in tandem with situs inversus totalis in the published literature. This rarity prompts us to report our case.

\section{Case presentation}

A 45-year-old Pakistani female who had been a known hypertensive for two years, presented to our out-patient department with the a few months history of intermittent bouts of left upper quadrant abdominal pain associated with nausea and flatulent dyspepsia. Fatty meal was reported in particular to precipitate the pain. There was no history of fever, jaundice, pruritis, clay colored stools or high colored urine. The physical examination was unremarkable except for apex beat palpable in the right fifth inter-costal space, raising the suspicion of dextrocardia. 
She was intensively investigated with laboratory and imaging investigations. Her liver function tests, serum amylase, blood complete tests, Serum urea, serum creatinine and urine examinations were all essentially normal. The abdominal ultrasonography revealed situ inversus with multiple gallstones in left sided gall bladder. The common bile duct was reportedly normal on the ultrasound scan. Chest X-ray revealed dextrocardia while echocardiography confirmed the dextrocardia with grade I diastolic dysfunction.

Until now neither the patient nor any of her family members were aware that she had situs inversus totalis. The patient and her husband were counseled about the diagnosis of symptomatic gall stones in the context of her congenital anomaly of visceral transposition. Having informed in detail about the pros and cons of surgical intervention, they preferred open cholecystectomy over laparoscopic cholecystectomy.

The abdomen was opened through a left standard subcostal incision. There was complete situs inversus viscerum with the hepatobiliary structures on the left side and spleen on the right side. The gastric fundus was on right side while duodenal first part was occupying the left hypochondrium. The appendix and caecum were found in the left iliac fossa.

Owing to dense adhesions in the Calot' triangle area, fundus-first technique of cholecystectomy was employed. Upon reaching the Calot's triangle, gall bladder was found distended with multiple calculi, and the common bile duct (CBD) was found to be grossly dilated, approximately $2 \mathrm{~cm}$ in diameter (see Figure 1). A per-operative cholangiogram was performed which showed fusiform dilatation of entire extra-hepatic biliary tree (see Figure 2). There was no stone in the CBD. The diagnosis of choledochal cyst type Ic was thus made. Cholecystectomy was completed and the choledochal cyst left alone for further detailed evaluation postoperatively and subsequent definitive management with excision and Roux-en-Y hepaticojejunostomy. The abdominal wound was closed in layers after placing a drain in the left subhepatic space. The patient's post-operative period was uneventful and the drain was removed after 48 hours. The patient was discharged home on 5th postoperative day. Histopathological examination of the resected surgical specimen revealed chronic cholecystitis and cholelithiasis. The patient was lost to further follow up and the planned definitive management of the choledochal cyst with excision and Roux-en-Y hepaticojejunostomy.

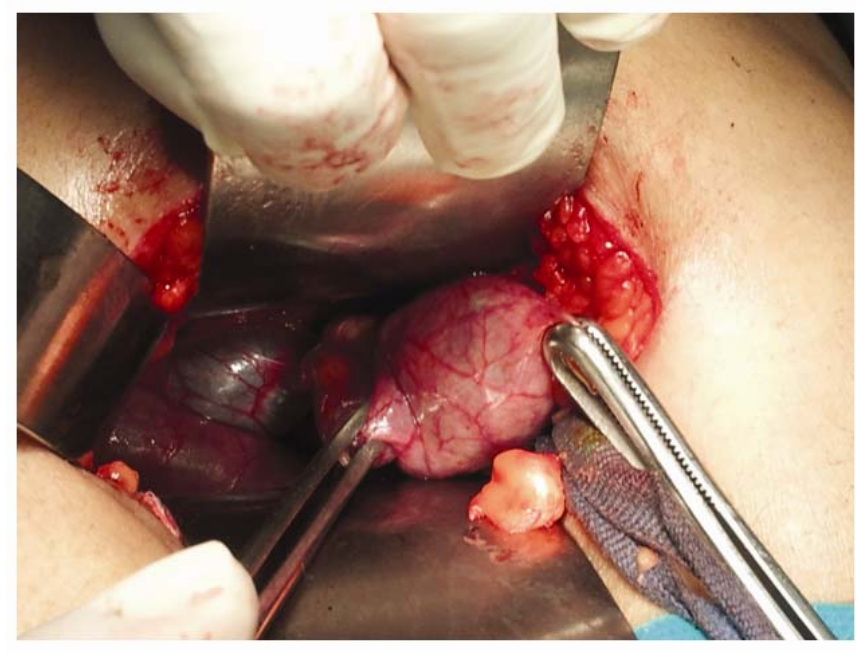

Figure 1. Intra-operative photograph showing left sided hepatobiliry tract with dilated common bile duct.

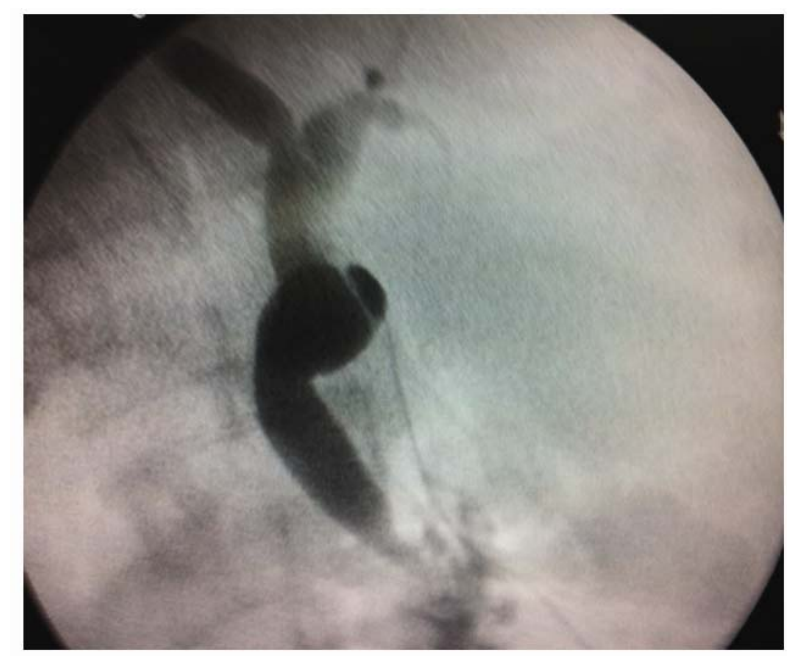

Figure 2. Per-operative cholangiogram showing the choledochal cyst.

\section{Discussion}

As was the case with our patient, the situs inversus abdominus is usually diagnosed incidentally when the patient undergoes some imaging investigation for an abdominal pathology. The variations from normal location of viscera pose 
diagnostic and management dilemmas when the patient develops some visceral pathology. The anomaly is thus attended by differences in clinical presentation, diagnostic elusiveness, and the need for modifications in operative techniques. Accurate imaging investigations of the viscera become imperative not only for making a correct diagnosis but also for choosing appropriate therapeutic interventions, surgical planning, and the type and location of surgical incision.

Our patient had choledochal cyst (CC) which on its own is even a more rarer anomaly than situs inversus abdominus. The estimated incidence of CCs varies from 1 in 13, $¥ 00$ to 1 in 2 million. They are far more common in Japan and Asia than in the west. The optimal treatment entails surgical excision to reduce risk of cholangiocarcinoma which develops in $9 \%-28 \%$ of the cases ${ }^{[6-8]}$. Todani $\mathrm{T}$ et al. have classified CCs into six types. The various types include: Type Ia- dilatation of extrahepatic duct; Type Ib- discrete segmental dilation of extrahepatic duct; Type Ic- fusiform dilation of entire choledochus; Type II diverticula of choledochus; Type III cyst/choledochocele - distal dilation of choledochus within duodenal wall; Type IVa- combined intrahepatic and extrahepatic duct cysts; Type IVb - multiple extrahepatic bile duct cysts and Type V/Caroli's disease- multiple intrahepatic bile duct cysts ${ }^{[8]}$. Type\#C is the most common type (80\%-90\%) and our patient had the same type of CC.

In our patient we undertook open cholecystectomy with slight modifications as opposed to the standard procedure performed in an otherwise normal individual. The left-sided open cholecystectomy required a mirror image incision to the conventional right-sided cholecystectomy. The procedure was essentially the same and was executed safely and careful attention was paid to avert the possibility of any iatrogenic injuries to the common bile duct, liver and gut. The operating time was almost doubled because of the altered orientation and ergonomic challenges encountered per operatively. The published literature shows a recent growing trend towards laparoscopic cholecystectomy for cholelithiasis patients with situs inversus totalis ${ }^{[9-13]}$.

Our case serves as reminder of the fact that in all such challenging patients the surgical planning should not rely solely on abdominal ultrasonography as it may fail to exactly delineate all the associated subtle pathologies, as we witnessed in our case. Rather one should keep a high index of suspicion for such anomalies and thoroughly investigate them through preoperative computerized tomography scan (CT scan) and magnetic resonance imaging (MRI). Though we did not perform in our case, it would have been even more useful if a magnetic resonance cholangio-pancreatography (MRCP) was performed, in order to exactly delineate the anomalous anatomy of the hepatobiliary tract, thus enabling better surgical planning, patient's preparation and counseling for definitive surgery for the CC. More invasive diagnostic techniques include percutaneous transhepatic cholangiography (PTC) and endoscopic retrograde cholangiopancreatography (ERCP) ${ }^{[6-9]}$.

There are two significant points in our case that merit due attention. First, the diagnosis of choledochal cyst was missed by the preliminary ultrasound scan. When faced with a rare anomaly of this type, one may lose attention to detail of individual structures and hence liable to miss another co-existing important pathology, as was the case with our patient. Second such anomalies have implications for the surgical team regarding performing surgery on mirror imaged reverse oriented gut and liver.

\section{Conclusions}

Individuals with situs inversus pose diagnostic and management dilemmas when they develop some abdominal pathology. Both ultrasonologists as well as surgical team members should be mindful of the possibility of an underlying choledochal cyst when they are dealing with symptomatic gall stone disease in such patients. This will ensure appropriate surgical planning and avert intra-operative management dilemmas.

\section{Consent}

Written informed consent was obtained from the patient for publication of this manuscript and any accompanying images. A copy of the written consent is available for review by the Editor-in-Chief of this journal. 


\section{References}

[1] Cleveland M: Situs in versus viscerum: anatomic study. Arch surg. 1926; 13(343).

[2] Cissé M, Touré AO, Konaté I, Dieng M, Ousmane Ka, Touré FB, et al. Appendicular peritonitis in situs inversus totalis: a case report. Journal of Medical Case Reports. 2010; 4: 134. http://dx.doi.org/10.1186/1752-1947-4-134

[3] Huang SM, Yao CC, Tsai TP, Hsu GW. Acute appendicitis in situs inversus totalis. J Am Coll Surg. 2008 ; $207: 954$. http://dx.doi.org/10.1016/j.jamcollsurg.2008.03.030

[4] Budhiraja S, Singh G, Miglani HP, Mitra SK: Neonatal intestinal obstruction with isolated levocardia. J Pediatr Surg. 2000; 35: 1115-6. http://dx.doi.org/10.1053/jpsu.2000.7839

[5] Karimi A, Omran AS, Ahmadi H, Yazdanifard P. Total myocardial revascularization for situs inversus totalis with dextrocardia: a case report. Journal of Medical Case Reports. 2007; 1: 18. http://dx.doi.org/10.1186/1752-1947-1-18

[6] Harun SM, Abdullah BJJ, Rajasingam V. What cyst is this? Biomed. Imaging Interv J. 2007; 3: e44. http://dx.doi.org/10.2349/biij.3.4.e44

[7] Memel DS, Balfe DM, Semelka RC. The Biliary Tract. In: Joseph LKT, Stuart SS, Sagel RJ, Heiken JP, editors. Computed body tomography with MRI correlation. 3rd ed. Philadelphia, NewYork: Lippincott-Raven Publishers. 1998: 779-844.

[8] Todani T, Watanabe Y, Narusue M, Tabuchi K, Okajima K. Congenital bile duct cysts: Classification, operative procedures, and review of thirty-seven cases including cancer arising from choledochal cyst. Am J Surg. 1977; 134: 263-269. http://dx.doi.org/10.1016/0002-9610(77)90359-2

[9] Kumar S, Fusai G. Laparoscopic cholecystectomy in situs inversus totalis with left-sided gall bladder. Ann R Coll Surg Engl. 2007; 89(2): W16-8. http://dx.doi.org/10.1308/147870807X160461

[10] de Campos Martins MVD, Falcão JLP, Skinovsky J,de Faria GMSS. Single-port cholecystectomy in a patient with situs inversus totalis presenting with cholelithiasis: a case report. Journal of Medical Case Reports. 2012; 6: 96. http://dx.doi.org/10.1186/1752-1947-6-96

[11] Kamitani S, Tsutamoto Y, Hanasawa K, Tani T. Laparoscopic cholecystectomy in situs inversus totalis with "inferior" cystic artery: a case report. World J Gastroenterol. 2005; 11: 5232-4.

[12] Pitakoudis M, Tsaroucha AK, Katomichelakis M, Polychronidis A, Simopoulos C. Laparoscopic cholecystectomy in a patient with situs inversus using ultrasonically actived coagulating scissors. Report of a case and review of the literature. Acta Chir Belg. 2005; 105: 114-7.

[13] Damian Mckay D, Blake G. Laparoscopic cholecystectomy in situs inversus totalis: a case report. BMC Surg. 2005; 5: 5. PMCID: PMC 555757. 